\title{
Surveillance, Fire Detection and Protection System for Substations
}

\author{
Athul Satheesh \\ U G Student \\ Department of Electrical and Electronics Engineering \\ Mar Baselios College of Engineering and Technology \\ Thiruvananthapuram, Kerala, India

\section{R. S Praveen Raj \\ Assistant Professor} \\ Department of Electrical and Electronics Engineering \\ Mar Baselios College of Engineering and Technology \\ Thiruvananthapuram, Kerala, India
}

\author{
Govind P N \\ U G Student \\ Department of Electrical and Electronics Engineering \\ Mar Baselios College of Engineering and Technology \\ Thiruvananthapuram, Kerala, India \\ Harinarayanan S M \\ U G Student \\ Department of Electrical and Electronics Engineering \\ Mar Baselios College of Engineering and Technology \\ Thiruvananthapuram, Kerala, India
}

\author{
Mukul Krishna \\ U G Student \\ Department of Electrical and Electronics Engineering \\ Mar Baselios College of Engineering and Technology \\ Thiruvananthapuram, Kerala, India
}

\begin{abstract}
Fire detection is most important in substations, due to its dangerous nature. It is necessary to detect fire early with accuracy. There comes the need of an accurate system for fire detection. So with the help of Neural Network we can detect fire from a surveillance video. The older sensor based systems and image processing based methods are not accurate and they often give false alarms due to wrong detection in outdoor areas. They need high implementation cost and not easy to cover larger areas. The environmental changes can also affect them. They have low performance and cause false alarms while there are shadows, varying lights and fire coloured objects. Neural Network helps to improve the fire detection capability with increased accuracy. It can detect fire in multiple situations as like day and night.

To provide a proper fire detection in substation yard, Neural network based fire detection is implemented. This system consists of a video camera which takes live footages of the area. The video is processed by the Neural Network on the basis of fire detection algorithm. The network is trained with different models and different settings to understand the nature of fire which is similar to the GoogLeNet. Various images of fire are given as input to train the network using ImageNet. In order to prevent false alarm fire like substances are taught to the network, which improves accuracy. As the no: of image sets used for training the network increases, the accuracy of the networks increases. Once the network is trained it will be able to detect fire and give alerts accurately. Compared to traditional fire detection methods neural networks provide accurate and fast response to fire in both indoor and outdoor conditions.
\end{abstract}

Index Terms - Neural Network, sensor, fire detection, accuracy.

\section{INTRODUCTION}

There are so many natural and unnatural disasters which may cause harm to humans and other living things. Of these fire is one of the major threat, which may cause huge damages [6]. Forest fire, short circuits and other situations creates large amount of fire and explosions. The major reasons of fire accidents are due to malfunctions of electrical equipment and wiring, improper handling of flammable materials, human errors and carelessness. The delayed actions and prevention against fire accidents may lead to large economic losses and even deaths to many. Older sensor based systems needs close proximity for detection and preventive measures like water spraying [3]. Then some more accurate systems like optical sensors to detect light and flame came [19]. Advanced techniques like image processing [7], pattern recognition [8][9], transfer learning [20], computer vision [13] [9], neural network [1] etc. made it possible to detect images with most accuracy from images and even videos now. Thus CNN based fire detection [1][2] provides a cost effective and accurate fire detection and it even gives possibilities to early stage prediction of fire [18] which helps to prevent forest fires.

\section{A. Problem}

Currently there is no fire protection system for substation yards. Even though protective devices like relays, circuit breakers are present, but there are chances of short-circuit and fire accidents. It is necessary for substations dealing with high voltage to have an accurate fire detection system.

\section{B. Solution}

Convolutional Neural Network based fire detection provides the way with surveillance and fire detection for electrical substations [1]. It uses the features like colour [12], shape, pattern [8], disorder nature [14] etc. to extract the features of fire from a video. The video is converted into each picture frame [11] and then it is further reduced 
into pixels for comparing the patterns [8] with already trained details of fire [1][5]. It uses surveillance video camera to continuously monitor the substation yard and this video is fed to the neural network trained system which compares it with trained data's and gives output as fire or non-fire [1] [10].

\section{METHODOLOGY}

\section{A. $\quad$ Phases}

\section{Phase-1}

Creating a neural network model with various datasets of fire and non-fire images and videos.

Phase-2

Training of system with given datasets and integration of sensors with control unit.

Phase-3

Testing of created model with different datasets and determination of accuracy of the system.

\section{B. Implementation}

The substation mainly consists of two plots.

1. Outer yard.

2. Control Centre

Currently there is no fire detection techniques used in outer yards. Even though sensors are placed, it is not sure that they detect fire with greater accuracy. It is not good to implement sensors with high voltage equipment. It is not practical to implement fire detection using heat/smoke sensors in outdoor yard of substations [2]. So we prefer $\mathrm{CNN}$ based fire detection on substation yards. Heat and smoke sensors are placed in control room of substations to detect fire [3]. A screen will show live status of the monitoring area as there is fire or not

[15] and an alarm will be initiated on detecting the fire [16] and a WhatsApp message will be sent to the respective person with help of active internet connection [4] [15]. Hence a proper fire detection and protection system is achieved.

\section{MOTIVATION}

- The absence of a proper fire detection system in substations.

- Drawbacks of older sensor based systems

- Increasing fire accidents due to short circuit.

\section{BLOCK DIAGRAM}

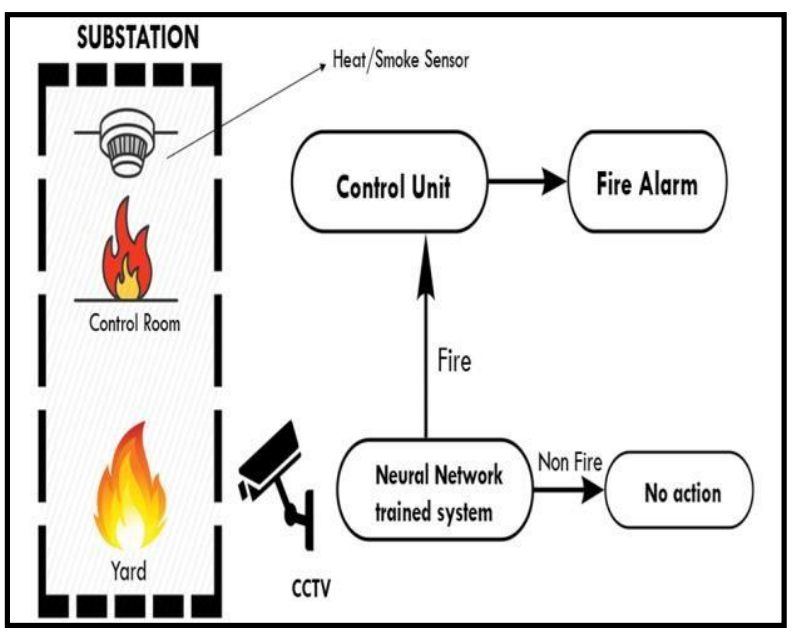

A.

\section{Explanation}

The CCTV continuously monitors the substation yard and the video is fed to the neural network trained system [1][2]. At the same time the heat/smoke sensors are monitoring change of heat in control room. When there is a fire the neural network will identify it and gives signal to control unit [15]. If there is fire the control unit will trigger the alarm [16]. And if the heat sensors are activated they also will give signal to control unit and alarm will go on. The control unit will give alarm and warnings so the person there can do necessary actions like switching off power supply, isolating area, seeking external [5] help etc.

\section{B. Circuit of Heat/Smoke Sensor}

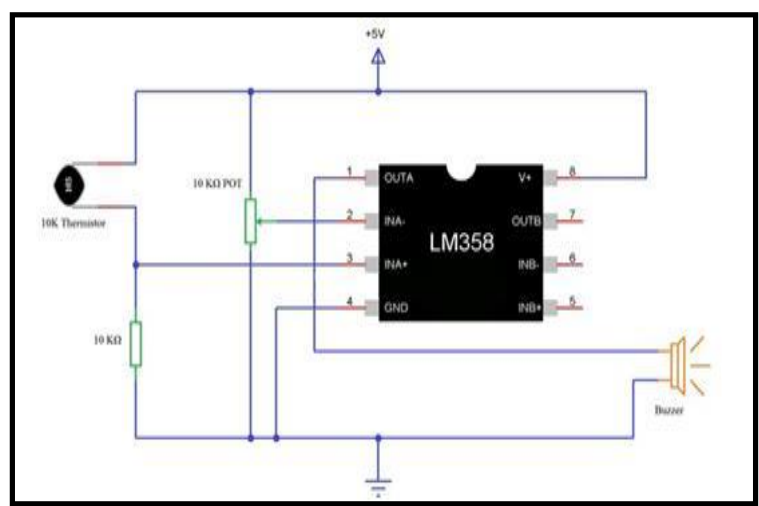


The circuit consists of an operational amplifier IC and a thermistor. The thermistor will act as a temperature sensor and when there is a change in temperature it will reduce the resistance of the thermistor to cause a low resistance path and will trigger the buzzer to make a warning alarm.

\section{COMPONENTS}

\section{HARDWARE}

A. $C C T V$

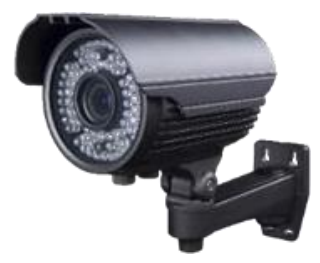

CCTV (closed-circuit television) may be a TV system within which signals don't seem to be in public distributed however area unit monitored, primarily for police work and security functions. There area unit a spread of CCTV cameras supported the applications. They embrace wide angle to a tele vary CCTV that has coverage of kilometers of space. Even visual sense recording is feasible in CCTV cameras.

\section{B. LM 358 IC}
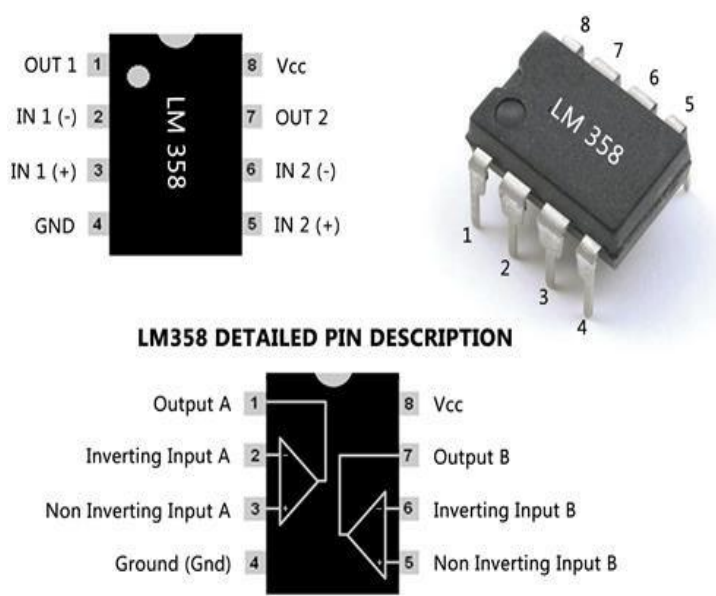

The LM358 IC is a great, low power and easy to use dual channel op-amp IC. This IC is designed for specially to operate from a single power supply over a wide range of voltages. LM358 can be used as transducer amplifier.

\section{Thermistor}

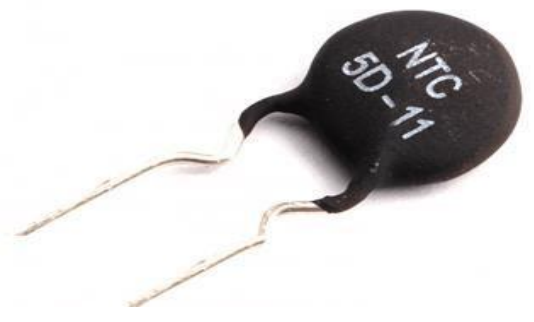

A thermistor is a temperature sensitive resistor; they are often used as a temperature sensor. An NTC (Negative Temperature Coefficient) thermistor is a thermally sensitive resistor whose resistance exhibits a large, precise and predictable decrease as the core temperature of the resistor increases over the operating temperature range. The temperature and resistance of thermistor are inversely proportional, that is as temperature increases the resistance of thermistor decreases and circuit will be closed and alarm will go on.

\section{Resistor}

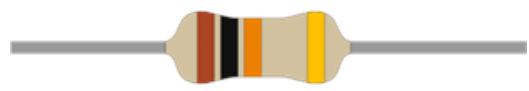

A resistor is a passive two-terminal electrical part that implements impedance as a circuit component. In electronic circuits, resistors scale back current flow, change signal levels, to divide voltages.

Square measure fastened and variable resistors used as per the necessity.

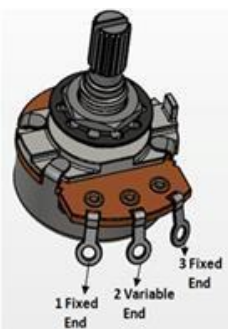

Here variable resistor is used to control the sensitivity of the thermistor. By varying the sensitivity, we can control the range of heat detection of the temperature sensor.

E. Buzzer

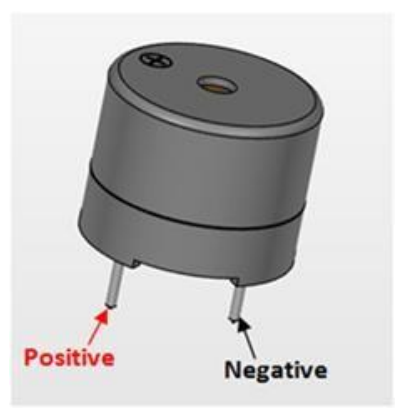


A buzzer is a tiny low nonetheless economical element to feature sound options to our project/system. It's tiny and compact 2-pin structure therefore are often simply used on breadboard, Perf Board and even on PCBs that makes this a widely used element in most electronic applications.

\section{SOFTWARE}

Python is used for controlling the neural network in which we create Fire Net (same as that of Google Net) in which fire and non-fire data are being trained and identified. The OpenCV and TensorFlow are the libraries used for the training and identification.

\section{A. Python}

Python is an interpreted, high-level, general-purpose programming language. Created by Guido van Rossum and first released in 1991, Python's design philosophy emphasizes code readability with its notable use of serious whitespace. Its language constructs and objectoriented approach aim to assist programmers write clear, logical code for little and largescale projects.[1][2] Python is dynamically typed and garbage-collected. It supports multiple programming paradigms, including structured (particularly, procedural), object-oriented, and functional programming. Python is often described as a "batteries included language" due to its comprehensive standard library. Python was conceived within the late 1980s as a successor to the ABC language. Python 2.0, released in 2000, introduced features like list comprehensions and a garbage collection system capable of collecting reference cycles. Python 3.0, released in 2008, was a major revision of the language that is not completely backward compatible, and much Python 2 code does not run on Python 3 without certain modification. The Python 2 language was officially discontinued in 2020 (first planned for 2015), and "Python 2.7.18 is that the last Python

2.7 release and thus the last Python2 release." No more security patches or other improvements are going to be released for it. With Python 2's end-of-life, only Python

3.5.x and later are supported. Python interpreters are available for many operating systems. A global community of programmers develops and maintains CPython, an open source reference implementation. A non-profit organization, the Python Software Foundation, manages and directs resources for Python and C Python development.

\section{B. Open $c v$}

Open CV (Open Source Computer Vision Library) may be a library of programming functions mainly aimed at real-time computer vision. Originally developed by Intel, it was later supported by Willow Garage (which was later acquired by Intel). The library is cross-platform and free to be used under the open-source BSD license.

\section{Tensor flow}

TensorFlow may be a free and open-source software library for dataflow and differentiable programming across a range

of tasks. It is a symbolic math library, and is additionally used for machine learning applications like neural networks. It is used for both research and production at Google. With the online system called 'Twilio', the WhatsApp message is being sent to the corresponding number. Collection of datasets of fire for training neural network is done. Different images of fire were trained and the neural network model is being implemented. The fire detection used is CNN based fire detection. The neural network works just like a human being, by providing sufficient training it can become highly effective. From the data it gets from the CCTV, the neural network decides whether there is fire in the yard or not from the trained data. If there is no fire detected, it does not take any action. If the fire is detected, the signal is sent to control unit, where an alarm rings and a warning message is sent to corresponding WhatsApp numbers.

\section{RESULT}

It was found that the camera was able to detect the distance which was not possible for normal sensors. The sensor ability was measured by showing the light from the matchstick towards the sensor and the sensor detected it as fire and the alarm rang.
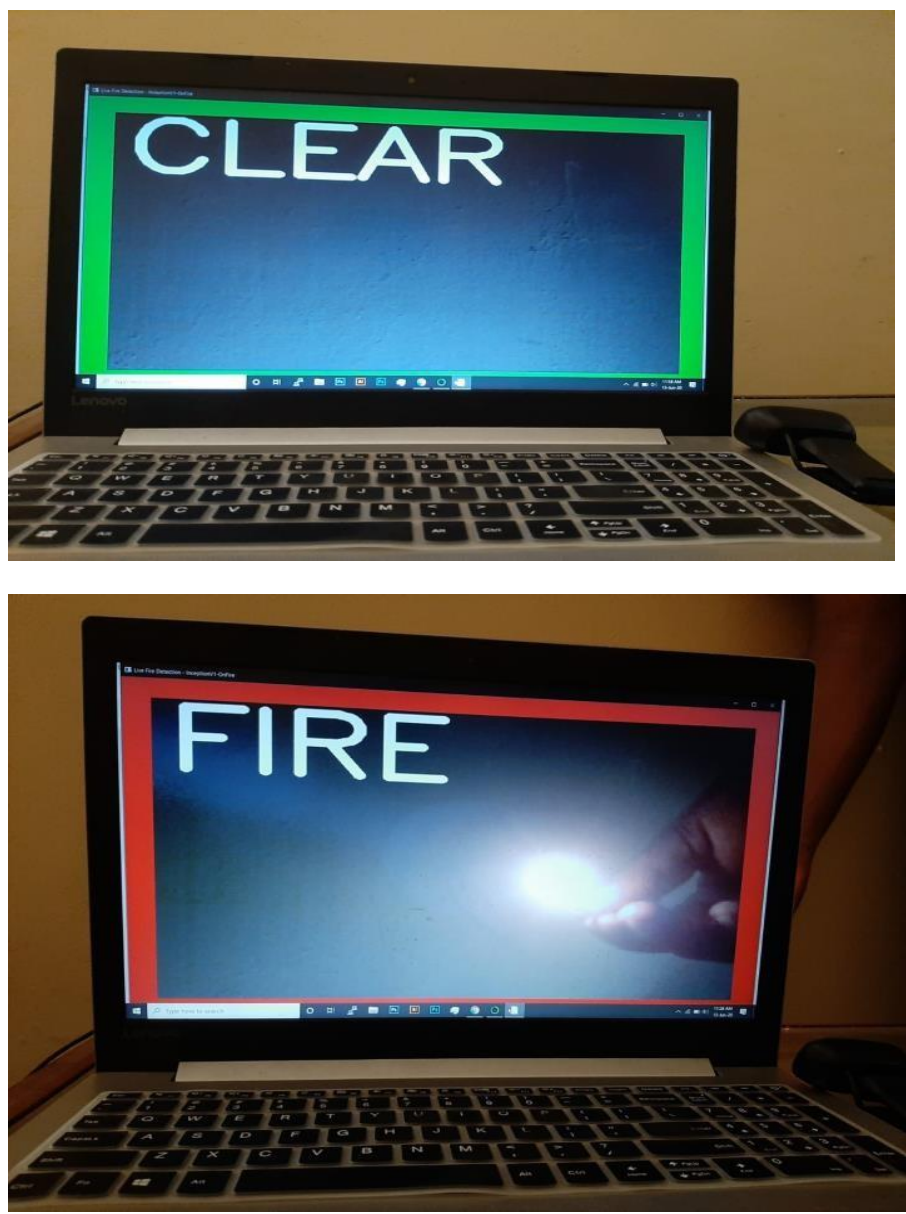


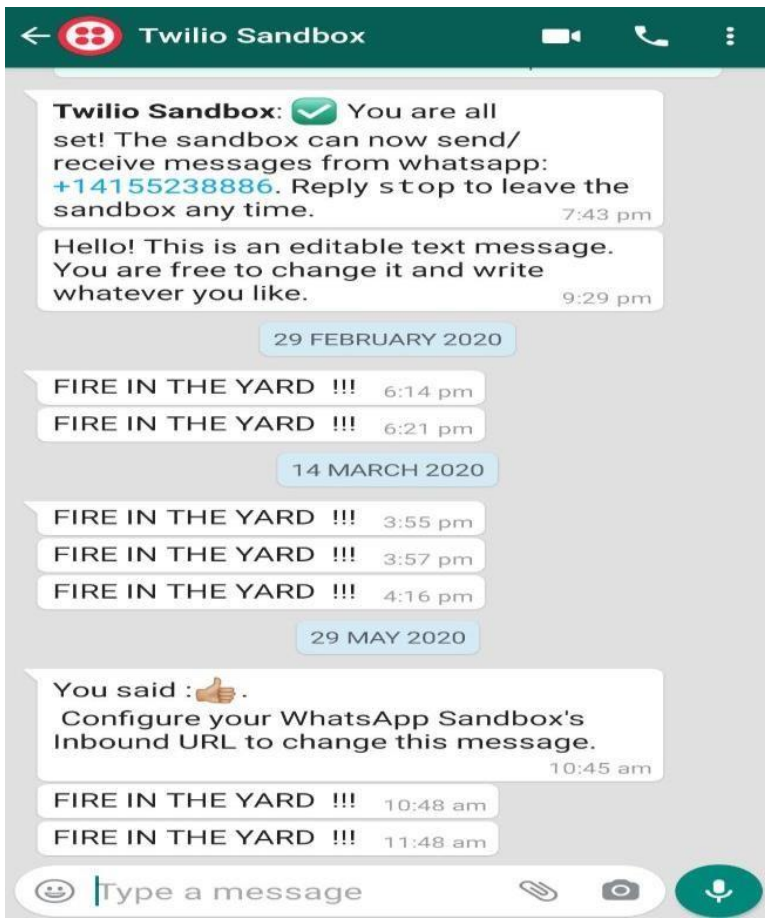

\section{CONCLUSION}

The existing system do not provide any mechanism for fire detection and protection on substation yards. Mostly substations are dealing with high voltage $\mathrm{AC}$ currents which are highly dangerous in nature and chances of a short circuit are very high. So we introduced Neural Network based fire detection system which is based on Artificial Intelligence to detect fire with more accuracy [1][2]. This system detects fire from a surveillance video taken from outside and sensors monitors presence of fire in inside control room and if fire is present an alarm is initiated [16] and a caution message is sent to the respective WhatsApp number [4] [15]. Since the neural network is trained with a numerous images and videos of fire and non-fire data the result obtained from final model will be more accurate and fast. Hence by the integration of convolutional neural network with sensor systems will be able to cover all areas of a substation for detecting fire with more accuracy and safety.

\section{REFERENCES}

[1] Khan Muhammad, J Ahmad, Mehmood, Seungmin, Sung Wook Baik, "Convolutional neural networks based fire detection in surveillance videos," IEEE Access, vol.6, no. , pp. 18174 18183, 2018.

[2] Khan Muhammad J Ahmad, and S.W. Baik, "Early Fire Detection using Convolutional Neural Networks during Surveillance for Effective Disaster Management," Neurocomputing, 2017/12/29/ 2017.

[3] K. Muhammad, R. Hamza, J. Ahmad, J. Lloret, H. H. G. Wang, and S. W. Baik, "Secure Surveillance Framework for IoT systems using Probabilistic Image Encryption," IEEE Transactions on Industrial Informatics, vol. PP, pp. 1-1, 2018

[4] Noorinder, Jaspreet Singh, Ekambir Sindhu, "Raspberry Pi based Smart Fire Management System employing Sensor based Automatic Water Sprinkler," 2017 InternationalConference on Power and Embedded Drive Control (ICPEDC) .

[5] K. Muhammad, J. Ahmad, and S. W. Baik, "Early Fire Detection using Convolutional Neural Networks during Surveillance for Effective Disaster Management," Neurocomputing, 2017/12/29/ 2017

[6] H. J. G. Haynes, "Fire Loss in the United States During 2015 http://www.nfpa.org/, 2016.

[7] T.-H. Chen, P.-H. Wu, and Y.-C. Chiou, "An early fire-detection method based on image processing," in Image Processing, 2004. ICIP'04. 2004 International Conference on, 2004, pp. 1707-1710.

[8] C.-B. Liu and N. Ahuja, "Vision based fire detection," in Pattern Recognition," 2004. ICPR 2004. Proceedings of the 17th International Conference on, 2004, pp. 134-137.

[9] B. U. Töreyin, Y. Dedeoğlu, U. Güdükbay, and A. E. Cetin, "Computer vision based method for real-time fire and flame detection," Pattern recognition letters, vol. 27, pp. 49-58, 2006.

[10] J. Choi and J. Y. Choi, "Patch-based fire detection with online outlier learning," in Advanced Video and Signal Based Surveillance (AVSS), 2015 12th IEEE International Conference on, 2015, pp. 1-6.

[11] G. Marbach, M. Loepfe, and T. Brupbacher, "An image processing technique for fire detection in video images," Fire safety journal, vol. 41, pp. 285-289, 2006

[12] T. Celik and H. Demirel, "Fire detection in video sequences using a generic color model," Fire Safety Journal, vol. 44, pp. 147-158, 2009.

[13] P. V. K. Borges and E. Izquierdo, "A probabilistic approach for vision- based fire detection in videos," IEEE transactions on circuits and systems for video technology, vol. 20, pp. 721-731, 2010.

[14] A. Rafiee, R. Dianat, M. Jamshidi, R. Tavakoli, and S. Abbaspour, "Fire and smoke detection using wavelet analysis and disorder characteristics," in Computer Research and Development (ICCRD), 2011 3rd International Conference on, 2011, pp. 262265

[15] S. Bayoumi, E. AlSobky, M. Almohsin, M. Altwaim, M. Alkaldi and M.Alkahtani, "A Real-Time Fire Detection and Notification System Basedon Computer Vision," 2013 International Conference on IT Convergenceand Security (ICITCS), Macao, pp. 1-4, 2013.doi:10.1109/ICITCS.2013.6717783.

[16] M. S. Bin Bahrudin, R. A. Kassim and N. Buniyamin, "Development of Fire alarm system using Raspberry Pi and Arduino Uno," 2013 International Conference on Electrical, Electronics and System Engineering (ICEESE), Kuala Lumpur, pp. 43-48, 2013. doi: 10.1109/ICEESE.2013.6895040.

[17] J. Yang, B. Jiang, B. Li, K. Tian, and Z. Lv, "A fast image retrieval method designed for network big data," IEEE Transactions on Industrial Informatics, 2017.

[18] S. Verstockt, T. Beji, P. De Potter, S. Van Hoecke, B. Sette, B. Merci, et al., "Video driven fire spread forecasting (f) using multi-modal LWIR and visual flame and smoke data," Pattern Recognition Letters, vol. 34, pp. 62-69, 2013.

[19] D.Starikov, C. Boney, R. Pillai and A. Bensaoula, "Dual-band UV/IR optical sensors for fire and flame detection and target recognition," IEEE Sensors for Industry Conference, 2004 Proceedings, pp. 36-40, $2014 . \quad$ doi: 10.1109/SFICON.2004.1287124.

[20] S. J. Pan and Q. Yang, "A survey on transfer learning," IEEE Trans. Knowl. Data Eng., vol. 22, no. 10, pp. 1345-1359, Oct. 2010 